\title{
Terapia com surfactante pulmonar exógeno - o que é estabelecido e o que necessitamos determinar
}

\section{Exogenous surfactant therapy - what is established and what still needs to be determined}

\author{
Celso M. Rebello ${ }^{1}$, Renata S. M. Proença ${ }^{2}$, Eduardo J. Troster ${ }^{3}$, Alan H. Jobe ${ }^{4}$
}

\section{Resumo}

Objetivo: revisar os aspectos já consagrados da terapêutica com surfactante exógeno, e discutir o estágio atual da pesquisa onde ainda não existe consenso.

Fonte dos dados: revisão bibliográfica, utilizando o banco de dados Medline e Cochrane Database Library, associada à experiência dos autores em relação à terapêutica de reposição do surfactante exógeno.

Síntese dos dados: os aspectos principais em relação às características do surfactante: composição, pool, metabolismo, inativação e efeitos imediatos após sua administração já estão bem estabelecidos. Mas ainda permanecem diversas dúvidas em relação ao uso do surfactante exógeno, que ainda é preciso determinar, como a escolha do tipo de surfactante, o momento mais apropriado para o tratamento, a dose e o número de doses, a melhor técnica de administração e as complicações associadas ao seu uso. Atualmente, a pesquisa no campo da terapêutica com surfactante exógeno concentra-se em duas linhas principais: o uso do surfactante em outras patologias que não a síndrome de desconforto respiratório do recémnascido, e o desenvolvimento de novos surfactantes através da adição de proteínas, ou análogos de proteínas, com o objetivo de melhorar sua ação e de reduzir sua inativação por proteínas plasmáticas.

Conclusões: hoje, a utilização do surfactante exógeno tornou-se rotina dentro das unidades de terapia intensiva neonatais, mas permanece aberto um campo de pesquisa tanto em nível experimental como clínico.

J Pediatr (Rio J) 2002; 78 (Supl.2): S215-S226:surfactante, recém-nascido, síndrome do desconforto respiratório.

1. Doutor em Pediatria pela FMUSP. Coordenador da Unidade de Pesquisa Experimental do Instituto da Criança do Hospital das Clínicas da FMUSP. Médico da Unidade Neonatal do Hospital Israelita Albert Einstein.

2. Médica Assistente da Unidade de Pesquisa Experimental do Instituto da Criança do Hospital das Clínicas da FMUSP.

3. Doutor em Pediatria pela FMUSP. Coordenador do Centro de Terapia Intensiva Pediátrico do Hospital Israelita Albert Einstein. Coordenador do Centro de Terapia Intensiva do Instituto da Criança do Hospital das Clínicas da Faculdade de Medicina da Universidade de São Paulo.

4. Professor de Pediatria, Cincinnati Children's Hospital Medical Center.

\begin{abstract}
Objective: to review well-known aspects of exogenous surfactant therapy, and to discuss controversial points regarding the current state of research.
\end{abstract}

Sources: review of the literature, using Medline and Cochrane Database Library, in association with the authors' experience in relation to exogenous surfactant replacement therapy.

Summary of the findings: the main aspects of surfactant characteristics: composition, pool, metabolism, inactivation and immediate effects after its administration are well established. However, there are some doubts related to the use of exogenous surfactants that need to be cleared up: choice of surfactant type, most appropriate length of treatment, adequate dose and number of doses, best administration route and complications associated with its use. Currently, the research about exogenous surfactant therapy focuses on two aspects: the use of surfactant in other pathologies besides the respiratory distress syndrome of the newborn, and the development of new surfactants through the addition of proteins or analogous proteins, with the aim of improving its action and reducing its inactivation.

Conclusions: the use of exogenous surfactant has become a routine in neonatal intensive care units, but both clinical and experimental research is still necessary.

J Pediatr (Rio J) 2002; 78 (Supl.2): S215-S226: surfactant, newborn, respiratory distress syndrome.

\section{Introdução}

A história da terapêutica de reposição com surfactante exógeno se inicia com o fisiologista Neergaard, que, em 1929, escreveu, em alemão, "Novas noções em um princípio fundamental da mecânica respiratória: a força retrátil do pulmão, dependente da tensão superficial do alvéolo"1 . 
Demonstrando que o pulmão inflado com ar tinha uma pressão transpulmonar maior do que o pulmão inflado com o mesmo volume de água, ele especulou que a atelectasia do recém-nascido poderia ser causada "por consideráveis forças retráteis da tensão superficial nos pulmões".

Na década de 40, Gruenwald descreveu, estudando pulmões de recém-nascidos autopsiados, a clássica hipoaeração, levando os pulmões a uma semelhança com um órgão maciço, que poderia ser inflado com líquido, mas perdia a arquitetura normal quando inflado com $\mathrm{ar}^{2}$.

No final da década de 50, Avery e Mead propuseram que a deficiência de surfactante pulmonar presente em prematuros determinava o colabamento alveolar, sendo fundamental na patogênese da membrana hialina ${ }^{3}$.

O novo salto nesta terapêutica ocorreu com Fugiwara, em 1980. Em um estudo não controlado, foi utilizado, pela primeira vez, surfactante por via intratraqueal em prematuros com síndrome do desconforto respiratório (SDR), com melhora evidente da oxigenação, mostrando a eficácia da nova terapêutica ${ }^{4}$.

A década de 80 permitiu que vários investigadores realizassem estudos de surfactantes de várias origens (humano, porcino, bovino, sintético) versus placebo, que culminaram na disponibilização do surfactante para uso clínico de rotina, no final da década de 80 e no início da década de 90.

Nos últimos 12 anos, vários estudos de grande porte foram realizados, com o objetivo de determinar o melhor esquema terapêutico, abordando o momento do tratamento (profilático vs. terapêutico), o tipo de surfactante (sintético vs. natural), a dose, entre outras questões. Esses estudos foram recentemente revisados por Roger Soll em uma série de meta-análises ${ }^{5-7}$.

Em anos mais recentes, a pesquisa no campo da terapêutica com surfactante exógeno se centrou em três focos principais: o primeiro sobre o uso do surfactante em outras patologias que não a SDR, incluindo síndrome de aspiração meconial (SAM), pneumonias, síndrome de desconforto respiratório do tipo agudo (SDRA), hérnia diafragmática congênita, entre outras; o segundo foco seria o estudo dos efeitos do recrutamento alveolar, realizado com aumento do pico de pressão inspiratória durante a administração do surfactante; e o último centrado no desenvolvimento de novos surfactantes, através da adição de proteínas sintéticas, ou análogos de proteínas, com o objetivo de melhorar sua ação e de reduzir sua inativação por proteínas plasmáticas.

Este artigo tem como objetivo revisar os aspectos já consagrados da terapêutica com surfactante exógeno, e discutir o estágio atual da pesquisa onde ainda não existe consenso sobre o uso de surfactante exógeno.

\section{Características do surfactante \\ Composição e pool do surfactante}

É bem determinado que a composição química do surfactante pulmonar é bastante semelhante entre as várias espécies de mamíferos $^{8}$, não havendo diferenças que justifiquem o uso de uma particular espécie animal, para a extração do surfactante pulmonar com a finalidade de uso terapêutico.

O surfactante é constituído, de modo geral, por lipídeos e proteínas. Cerca de 80 a $90 \%$ de sua composição em massa é composta por lípides, incluindo lípides neutros e fosfolípides.

Cerca de 70 a $80 \%$ dos fosfolípides consiste de fosfatidilcolina, presente em sua maioria na forma saturada ( $45 \%$ da massa do surfactante). $\mathrm{O}$ fosfatidilglicerol representa 5 a $10 \%$ (em massa), enquanto o fosfatidilinositol, fosfatidiletanolamina e a fosfatidilserina constituem menos que $10 \%$ do total de lípides. O colesterol e os ésteres do colesterol representam menos do que $5 \%$ do surfactante em massa, sendo que o seu papel não é bem conhecido até o momento, embora ele modifique a fluidez e a organização das membranas lipídicas.

A principal função dos fosfolípides é a de atuar como uma molécula que reduz a tensão superficial na interface arlíquido do interior do alvéolo. Esta característica única permite evitar o colabamento alveolar no final da expiração, quando as forças que causam o colapso alveolar estão maximizadas, sendo inversamente proporcionais ao quadrado do raio alveolar. No entanto, a fosfatidilcolina sozinha não é capaz de explicar as principais características do surfactante: estabilidade durante a compressão, rápida adsorção da subfase para a superfície alveolar e a capacidade de reorganizar a monocamada, quando dispersa em um ambiente aquoso. Na realidade, à $37^{\circ} \mathrm{C}$ a fosfatidilcolina assume a forma gel, passando para a forma "líquida" e dispersa a $41^{\circ} \mathrm{C}^{9}$. Portanto, ela depende dos outros componentes do surfactante para a formação de uma camada estável na superfície alveolar, quando em temperatura fisiológica.

As proteínas representam cerca de $10 \%$ da massa do surfactante, sendo reconhecidas quatro proteínas, SP-A, SP-B, SP-C e SP-D.

A SP-A é uma proteína hidrossolúvel, sendo a mais abundante proteína do surfactante, representando $5 \%$ de sua massa. É composta por um grupo de monômeros, de 26$\mathrm{KD}$, unidos por forte ligação covalente, resultando em uma molécula de $650 \mathrm{KD}$. Entre suas funções, se destaca um papel na defesa imune pulmonar, em decorrência da capacidade de se ligar a carboidratos, e por interagir com células imune pulmonares. A ausência de SP-A prejudica a eliminação, tanto de bactérias como de vírus dos pulmões, facilitando a disseminação sistêmica de infecções ${ }^{10}$. Entre os principais papéis da SP-A, ainda se destaca o mecanismo protetor do surfactante em relação à inibição de sua função pelas proteínas presentes no edema alveolar ${ }^{11}$. 
A SP-A parece influenciar no metabolismo do surfactante apenas nos estudos in vitro, pois em animais transgênicos com deficiência desta proteína, não há influência sobre o metabolismo ou a função do surfactante.

A SP-B é uma pequena proteína hidrofóbica de $18 \mathrm{KD}$, fundamental para a função do surfactante pulmonar, sendo sua ausência congênita incompatível com a vida ${ }^{12,13}$.

Entre suas funções, se destacam a formação e a organização da mielina tubular no interior do alvéolo ${ }^{14}$, além do papel facilitador na adsorção da fosfatidilcolina na temperatura fisiológica.

A SP-C também é um peptídeo hidrofóbico, que forma uma estrutura helicoidal rígida, de 4,2 KD. Embora tenha funções semelhantes à SP-B, particularmente permitindo a adsorção da fosfatidilcolina na temperatura fisiológica, sua deficiência congênita não resulta em morte por insuficiência respiratória, embora possa haver evolução para doença pulmonar intersticial familiar ${ }^{15}$.

Pode-se dizer que, de todos os seus componentes, os lípides SP-B e SP-C são os principais responsáveis pelas propriedades biofísicas e funcionais do surfactante pulmonar.

A SP-D é uma glicoproteína hidrossolúvel, formada por agregados de monômeros de $43 \mathrm{KD}$, resultando em multímeros de $560 \mathrm{KD}$. De forma semelhante, a SP-A não está presente nos preparados de surfactante de origem animal obtidos por processo de extração lipídica, e não tem atividade de redução da tensão superficial, parecendo determinar um papel na defesa antiinfecciosa do pulmão, por se ligar com uma variedade de complexos carboidratos e glicolípides, interagindo com a superfície de bactérias e outros microrganismos ${ }^{16}$.

Em relação à quantidade de surfactante presente no pulmão, sabe-se que ela se correlaciona de maneira logarítmica à superfície alveolar, em várias espécies animais ${ }^{17}$. Em humanos, um relato baseado em cinco lavagens subsegmentares em voluntários adultos permitiu uma estimativa de pool alveolar da ordem de $3 \mathrm{mg}$ de surfactante $/ \mathrm{kg}^{18}$. Esta estimativa foi confirmada em um estudo envolvendo 24 cadáveres, de 13 meses a 80 anos, no qual a estimativa do pool alveolar de surfactante foi de $4 \mathrm{mg} / \mathrm{kg}$, e do pulmão total foi de cerca de $56 \mathrm{mg} / \mathrm{kg}^{19}$.

A quantidade de surfactante pulmonar diminui com a idade, porém não de maneira significante. É interessante notar que prematuros com SDR possuem uma quantidade de surfactante na faixa de 1 a $5 \mathrm{mg} / \mathrm{kg}^{20}$, semelhante à observada em adultos, e cerca de dez vezes inferior à observada em recém-nascidos de termo, sendo bastante inferior à dose utilizada para tratamento da SDR do recémnascido. Isso mostra que a estrutura do pulmão prematuro, e talvez a presença de proteínas na luz alveolar, determinam a necessidade de um pool maior no momento do nascimento, como o encontrado no pulmão do recém-nascido de termo, para assegurar uma função adequada.

\section{Metabolismo do surfactante}

O surfactante é produzido no pneumócito II. Os fosfolípides e as proteínas SP-B e SP-C são sintetizados no retículo endoplasmático rugoso, onde são armazenados; inicialmente, no complexo de Golgi, e, posteriormente, nos corpos lamelares (Figura 1). Periodicamente, estes últimos são expulsos do pneumócito II, quando o surfactante é liberado para a luz alveolar, organizando a mielina tubular.

A cinética da síntese e secreção para o interior do alvéoloé muito lenta, atingindo de 30 a 48 horas em animais recém-nascidos ${ }^{21}, \mathrm{e}$, de modo geral, este tempo é maior em recém-nascidos, quando comparado com adultos.

Após a secreção para o interior do alvéolo, o surfactante passa por um complexo ciclo (Figura 1). Inicialmente, as moléculas de gordura se organizam (particularmente com ajuda das proteínas), para formar a monocamada que reveste a superfície alveolar, a mielina tubular. Com sucessivos movimentos de contração e estiramento, que ocorrem a cada ciclo respiratório, parte da mielina tubular se desorganiza e se desprende do filme principal, na forma de pequenas vesículas, que são reabsorvidas para o interior do pneumócito II. Dentro da célula, uma pequena parte é catabolizada, enquanto que a maior parte do surfactante que é reabsorvido é misturada aos corpos lamelares, nos quais é reorganizado, num processo de reciclagem. Assim, nos prematuros, cerca de 50\% do pool alveolar é composto de surfactante com boa capacidade de reduzir a tensão superficial, e 50\% é composto por vesículas inativas a serem recicladas. Esta relação está mais desfavorável em situações de injúria pulmonar ${ }^{22}$.

Este processo de reciclagem minimiza a necessidade de síntese de surfactante, enquanto mantém um pool alveolar adequado, ao mesmo tempo em que ativa os componentes do surfactante, reabsorvidos para o pneumócito II através da adição de novos elementos (particularmente proteínas) e da reorganização estrutural dos lípides e proteínas. Este último processo é de particular importância no tratamento com surfactante exógeno, que passa a ser adicionado de SPA e SP-D (ausentes nos preparados comerciais) através da reciclagem.

Ainda em relação ao surfactante exógeno administrado para tratamento da SDR, foi observado que, rapidamente, este é incorporado ao tecido pulmonar, sendo recuperado apenas $40 \%$ no espaço alveolar, logo após a administração 23 .

O tratamento com surfactante exógeno não interfere com as vias metabólicas do surfactante endógeno, não havendo inibição por feed-back da sua produção.

As proteínas hidrossolúveis SP-A e SP-D são sintetizadas e liberadas de maneira independente dos fosfolípides e das proteínas lipossolúveis SP-B e SP-C.

\section{Inativação do surfactante}

O surfactante pode ter sua função de redução da tensão superficial alveolar inibida pelas proteínas plasmáticas que 


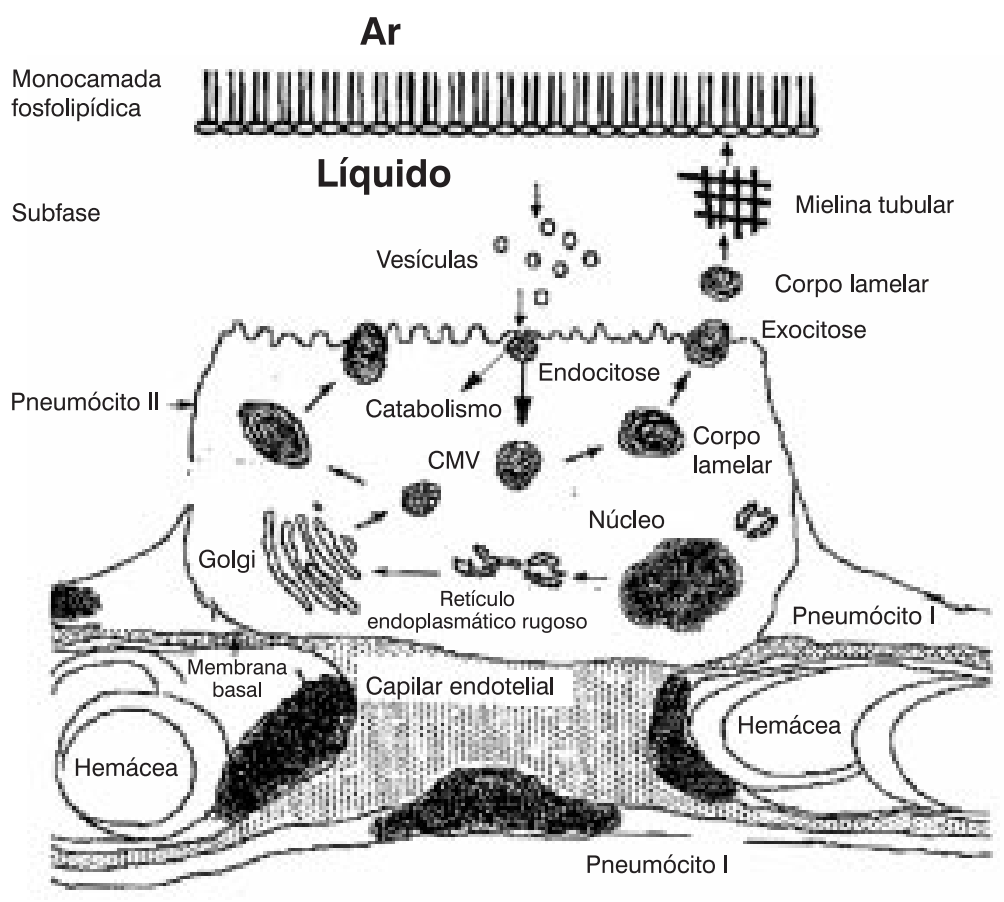

Modificado de Ikegami M, et al. Seminars in Perinatol 1993; 17:4.

Figura 1 - Esquema do metabolismo intracelular do surfactante

invadem o espaço alveolar na lesão pulmonar aguda. Esta inativação é um fenômeno reversível e ocorre, primariamente, devido a uma interferência na formação da monocamada de surfactante, causada pela presença de proteínas, através de um mecanismo de competição pela interface arlíquido.

O fenômeno inativação depende de vários fatores, incluindo a quantidade de surfactante e de proteínas que competem pela interface ar-líquido ${ }^{24}$, o tipo de proteína presente na luz alveolar, sendo que os monômeros de fibrina se situam entre as proteínas com maior capacidade de inativação do surfactante ${ }^{25}$, e da quantidade de proteínas específicas do surfactante (SP-A, SP-B e SP-C), que reduzem a inativação ${ }^{26}$. Este fenômeno é reduzido com o uso pré-natal de corticosteróides, presumidamente por um melhor mecanismo de reciclagem, resultando em maiores concentrações de proteínas específicas no produto final ${ }^{27}$.

\section{Efeitos imediatos do tratamento com surfactante}

A primeira resposta ao tratamento com surfactante é um rápido e intenso aumento na oxigenação, o que ocorre alguns minutos depois da administração, permitindo uma rápida redução nas concentrações de oxigênio inspirado.

A melhora da complacência ocorre mais lentamente, permitindo uma progressiva redução do pico máximo de pressão inspiratória utilizado, a fim de se manter um volume corrente adequado.

Este efeito pode ser melhor entendido observando-se a redução da pressão de abertura (pressão na qual o pulmão é preenchido acima do volume do espaço-morto) na fase inspiratória da curva pressão-volume de animais tratados com surfactante em comparação a animais controle ${ }^{28}$.

O maior recrutamento alveolar resulta em aumento do volume pulmonar máximo e maior estabilidade na expiração, quando os alvéolos conservam-se abertos, resultando em maior capacidade residual funcional. A somatória destes efeitos explica a dramática melhora na oxigenação observada após o tratamento com surfactante.

Do ponto de vista microscópico, o tratamento com surfactante leva a uma maior uniformidade na expansão alveolar, reduzindo as áreas de atelectasia/hiperdistensão, sendo um efeito da maior importância na redução da injúria pulmonar, obtida com o tratamento com surfactante ${ }^{29}$. 
Os efeitos sobre o fluxo sangüíneo pulmonar são controversos, tendo sido relatado aumento ${ }^{30}$ ou fluxo inalterado ${ }^{31}$ na artéria pulmonar.

\section{Surfactantes disponíveis e técnica de administração \\ Tipos de surfactante}

Existem duas escolhas básicas para a terapêutica com surfactante exógeno, que são: os surfactantes "naturais", obtidos de animais por um processo de extração lipídica de lavado ou homogenado pulmonar, que conserva SP-B e SPC em sua composição, com ou sem adição de fosfolípides ao produto final; e os surfactantes sintéticos, produzidos em laboratório. Uma descrição dos principais surfactantes disponíveis é feita na Tabela 1.

Embora ambas as classes de surfactante modifiquem o curso da SDR, as respostas, particularmente em curto prazo, são diferentes.
Os surfactantes naturais produzem uma resposta imediata na melhora da oxigenação e da função pulmonar, exigindo inclusive uma monitoração próxima e constante do recém-nascido, logo após o tratamento, a fim de se evitar complicações indesejáveis. Já os sintéticos demoram algumas horas para determinar os mesmos efeitos. A explicação deste fenômeno provavelmente está relacionada ao fato de que o surfactante sintético tem como função principal aumentar o pool alveolar e tecidual, que será reciclado no interior do pneumócito II, sendo adicionado a proteínas específicas, que não estão presentes no produto comercial.

Uma meta-análise comparou, recentemente, os estudos realizados com extratos de surfactante natural versus sintético, para o tratamento da SDR, porém nenhum utilizou os novos surfactantes sintéticos (KL-4 e rSP-C). A revisão dos resultados de 11 estudos demonstrou que ambas as classes de surfactantes mostraram-se efetivas para o tratamento da SDR, porém com um menor risco de pneumotórax e uma menor mortalidade associada ao tratamento com surfactan-

Tabela 1 - Principais surfactantes disponíveis para uso clínico ou experimental

\begin{tabular}{|c|c|c|c|}
\hline Nome comercial & Nome genérico & Preparação & Laboratório \\
\hline Surfacten & Surfactante-TA & $\begin{array}{l}\text { Extrato de pulmão bovino com adição } \\
\text { de DPPC, tripalmitoilglicerol e ácido palmítico }\end{array}$ & $\begin{array}{l}\text { Tokyo Tanabe } \\
\text { (Japão) }\end{array}$ \\
\hline Survanta & Beractant & $\begin{array}{l}\text { Extrato de pulmão bovino com adição } \\
\text { de DPPC, tripalmitoilglicerol e ácido palmítico }\end{array}$ & $\begin{array}{l}\text { Ross Products Division of } \\
\text { Abott Laboratories (EUA) }\end{array}$ \\
\hline Curosurf & Poractant & $\begin{array}{l}\text { Pulmão suíno submetido à extração com } \\
\text { clorofórmio-metanol; purificado por } \\
\text { cromatografia líquido-gel }\end{array}$ & $\begin{array}{l}\text { Chiesi Pharmaceuticals } \\
\text { (Itália) }\end{array}$ \\
\hline Infasurf & $\begin{array}{l}\text { Calf lung } \\
\text { surfactant extract }\end{array}$ & $\begin{array}{l}\text { Extrato de lavado pulmonar de bezerro } \\
\text { submetido à extração com clorofómio-metanol }\end{array}$ & $\begin{array}{l}\text { Forrest Laboratories } \\
\text { (EUA) }\end{array}$ \\
\hline BLES & $\begin{array}{l}\text { Bovine lipid } \\
\text { extract surfactant }\end{array}$ & $\begin{array}{l}\text { Extrato de lavado pulmonar de vaca } \\
\text { submetido à extração com clorofórmio-metanol }\end{array}$ & $\begin{array}{l}\text { BLES Bioquimicals } \\
\text { (Canada) }\end{array}$ \\
\hline Alveofact & SF-RI 1 & $\begin{array}{l}\text { Extrato de lavado pulmonar de vaca } \\
\text { submetido à extração com clorofórmio-metanol }\end{array}$ & $\begin{array}{l}\text { Boehringer Inglheim } \\
\text { (Alemanha) }\end{array}$ \\
\hline Exosurf & $\begin{array}{l}\text { Colfosceril plamitate, } \\
\text { hexadecanol, tyloxapol }\end{array}$ & DPPC com $9 \%$ de hexadecanol e $6 \%$ de tyloxapol & $\begin{array}{l}\text { Burroughs-Wellcome Co. } \\
\text { (EUA e Inglaterra) }\end{array}$ \\
\hline Pneumactant* & $\begin{array}{l}\text { Composto expandido de } \\
\text { pulmão artificial (ALEC) }\end{array}$ & DPPC e PG na razão 7:3 & $\begin{array}{l}\text { Britania Pharmaceuticals } \\
\text { (Inglaterra) }\end{array}$ \\
\hline Surfaxin & Lucinactant & $\begin{array}{l}\text { Peptídeo sintetizado quimicamente combinado } \\
\text { com fosfolipídeos e ácido palmítico }\end{array}$ & $\begin{array}{l}\text { Discovery Laboratories } \\
\text { (EUA) }\end{array}$ \\
\hline Venticute & rSP-C Surfactant & $\begin{array}{l}\text { SP-C recombinante combinado com fosfolipídeos } \\
\text { e ácido palmítico }\end{array}$ & $\begin{array}{l}\text { Byk Gulden } \\
\text { (Alemanha) }\end{array}$ \\
\hline
\end{tabular}


te natural, assim como uma tendência a um menor risco de evolução para displasia broncopulmonar ou morte.

\section{Momento do tratamento}

Classicamente, se comparou o uso profilático com o uso terapêutico do surfactante, para o tratamento da SDR. O termo "profilático" pode causar alguma confusão, porém, na maioria dos estudos, foi utilizado significando tratamento dentro de um intervalo de tempo, pré-determinado pelos pesquisadores, geralmente na sala de reanimação.

A lógica do uso profilático se baseia na melhor distribuição do surfactante, quando administrado antes da primeira respiração, e na menor lesão pulmonar, resultando em menos edema alveolar e menor inativação por proteínas.

A lógica do uso terapêutico baseia-se no tratamento apenas de recém-nascidos que evoluíram para SDR, evitando-se tratamentos e possíveis complicações desnecessárias.

Outros estudos, comparado uso profilático e terapêutico, foram revisados em uma meta-análise recentemente atualizada ${ }^{32}$, sendo que em todos os estudos foram utilizados surfactantes naturais.

Essa meta-análise demonstrou uma redução no risco de pneumotórax, enfisema intersticial, mortalidade e displasia broncopulmonar, ou mortalidade associada ao tratamento profilático. O estudo sugere que, para cada 100 recémnascidos tratados profilaticamente, haverá uma redução de pneumotórax em 2 recém-nascidos e de 5 óbitos. Por outro lado, não ficou claro o possível papel atenuante do uso de corticóide pré-natal nesses resultados.

Outra abordagem terapêutica estudada foi o tratamento precoce (dentro das primeiras duas horas de vida), ou tardio (depois da segunda hora de vida) da SDR já estabelecida. Em uma meta-análise, incluindo quatro estudos (dois com surfactante sintético e dois com natural), foi demonstrada uma redução no risco de pneumotórax, enfisema intersticial, mortalidade pré-natal, doença pulmonar crônica e doença pulmonar crônica e morte com 36 semanas de idade gestacional corrigida. Com a realização do tratamento nas primeiras duas horas de vida, há uma tendência de redução do risco para displasia broncopulmonar, ou morte com 28 dias de vida 6 .

A análise dos resultados das duas abordagens mostra as vantagens do tratamento profilático ou terapêutico até duas horas de vida. Fica evidente que, quanto mais cedo for realizado o tratamento, melhores são os resultados.

Uma proposta terapêutica de bom senso é o tratamento dos casos com diagnóstico estabelecido de SDR, sendo este realizado o mais precocemente possível, de preferência nas duas primeiras horas de vida.

Estudos comparando o uso profilático com o uso terapêutico nas duas primeiras horas de vida podem trazer esclarecimentos sobre a melhor abordagem do momento do tratamento.

\section{Dose e técnica de administração}

Considerando-se que o pool alveolar de surfactante de prematuro com SDR não difere de modo significante do pool alveolar de adulto sem patologia respiratória ${ }^{19}$, evidenciando o papel fundamental da "qualidade do surfactante" (conteúdo protéico), da imaturidade estrutural pulmonar dos prematuros e o papel inativador do extravasamento de proteínas para a luz alveolar, que ocorre no pulmão prematuro imediatamente após o nascimento ${ }^{33}$, fica evidente a necessidade de estabelecer a dose adequada de surfactante exógeno, para reverter ou minimizar esta situação no pulmão prematuro.

Um dos primeiros estudos a abordar esta questão foi realizado por Ikegami et al., em $1980^{34}$. Utilizando cordeiros prematuros, com surfactante natural de ovelha (contendo todas as quatro proteínas: SP-A, SP-B, SP-Ce SP-D), em doses que variaram de 19,53, 64 e $173 \mathrm{mg} / \mathrm{kg}$, e através da análise in vitro, por determinação da tensão superficial do surfactante, utilizando-se uma balança de Wilhelmy, observou-se que a dose de $64 \mathrm{mg} / \mathrm{kg}$ de surfactante produziu piores resultados em relação à dose superior.

Esses resultados estão de acordo com a proposta de Clements e King, que sugerem que a quantidade teórica de surfactante necessária para cobrir a superfície alveolar seria de $1 \mathrm{mg}$ (surfactante em massa) por grama de pulmão, o que corresponderia, aproximadamente, a uma dose de surfactante de, no mínimo, 36,4 mg (lípides totais) por kg de peso corpóreo, ou 40mg de surfactante $/ \mathrm{kg}^{35}$.

A maior parte dos estudos clínicos utilizou a dose de $100 \mathrm{mg} / \mathrm{kg}$, com exceção dos estudos com Curosurf ${ }^{\circledR}$ (surfactante obtido por extrato de pulmão porcino), em que a dose utilizada foi de $200 \mathrm{mg} / \mathrm{kg}$, e na maior parte dos estudos com surfactante humano, foi utilizada a dose de $60 \mathrm{mg} / \mathrm{kg}$.

Fujiwara comparou a dose de $120 \mathrm{mg} / \mathrm{kg}$ com a dose de $60 \mathrm{mg} / \mathrm{kg}$, encontrando melhores resultados (menor necessidade de suporte ventilatório e redução na incidência de hemorragia intracraniana e de displasia broncopulmonar) com a dose de $120 \mathrm{mg} / \mathrm{kg}^{36}$.

É razoável se supor que, com base nas considerações técnicas da quantidade mínima de surfactante necessária para se revestir a superfície alveolar, ao lado dos estudos que comparam diferentes doses de surfactante, e frente aos conhecimentos hoje disponíveis, de que a inativação do surfactante alveolar (presente mesmo com quantidades adequadas de surfactante) é um fenômeno altamente dosedependente, a dose inicial de $100 \mathrm{mg} / \mathrm{kg}$ (que em uma fase inicial foi utilizada empiricamente) pode ser assumida como adequada para a maioria das situações clínicas. A exceção seria a situação em que já se espera uma grande inibição da função do surfactante exógeno (tratamento tardio, em que o edema alveolar já está instalado e é intenso, SAM, hemorragia pulmonar, etc.) na qual, em vista do efeito dose-dependente da inativação, a dose inicial de $200 \mathrm{mg} / \mathrm{kg}$ pode ser mais adequada. 
Uma segunda abordagem seria a utilização de múltiplas doses de surfactante, em relação ao tratamento com dose única, também com o objetivo de reverter a inativação.

Nesse sentido, uma meta-análise que reuniu os resultados de dois estudos, comparando dose única com múltiplas doses de surfactante natural $(100 \mathrm{mg} / \mathrm{kg})$, demonstrou que esta última abordagem resultou em uma melhora da oxigenação, com menor necessidade ventilatória, uma redução na incidência de pneumotórax e uma tendência de redução na mortalidade ${ }^{7}$. O autor não observou um aumento na incidência de complicações, associado ao uso de surfactante em múltiplas doses.

De modo geral, levando-se em conta o ciclo metabólico dentro do pneumócito tipo II, particularmente se a lesão pulmonar inicialé evitada, não se faz necessária a utilização de mais de uma dose de surfactante.

\section{Técnica de administração}

Várias técnicas de administração foram utilizadas, com resultados diferentes.

O uso de surfactante por nebulização foi afastado após várias tentativas, uma vez que, mesmo utilizando nebulização ultra-sônica, a quantidade se surfactante que atinge os alvéolos é bastante reduzida, com cerca de 7,6\% da dose de surfactante atingindo os pulmões ${ }^{37}$. No entanto, deve ser salientado que esta leva a uma distribuição mais homogênea do que a instilação tradicional. A eficiência da nebulização se correlaciona diretamente com a complacência e o índice de eficiência ventilatória após o tratamento. Esta ineficiência da técnica provavelmente está relacionada ao tamanho da partícula gerada pelo nebulizador, à perda de surfactante no circuito e à localização do nebulizador no circuito do ventilador.

A técnica de administração em bolo foi utilizada com base nos estudos experimentais, nos quais ela se mostrou eficaz. A instilação logo após o nascimento, e anterior à primeira respiração, resulta em uma distribuição uniforme de surfactante, com excelente resposta clínica ${ }^{38}$, enquanto que a administração após um período curto de ventilação resulta em uma distribuição menos uniforme. Por outro lado, devido à óbvia interferência do tratamento ao nascimento com as manobras de reanimação, recomenda-se que o tratamento seja feito após a estabilização do recémnascido, de preferência em UTI neonatal.

Recém-nascidos tratados com o tórax estável na posição horizontal têm uma distribuição comparável de surfactante em ambos pulmões ${ }^{39}$, não havendo diferença na eficácia, comparando-se a técnica de administração em duas ou quatro alíquotas ${ }^{40}$ com manobras de posicionamento torácico.

A técnica recomendada é a administração em alíquota única, com o recém-nascido estável, na posição horizontal, após adequado posicionamento da cânula traqueal. O sur- factante deve ser administrado com o circuito do ventilador fechado, a fim de se evitar perda de pressões das vias aéreas, que determinariam colapso pulmonar. $\mathrm{O}$ uso de adaptadores com porta lateral, ou cânula traqueal com porta injetora na extremidade permitem a administração do surfactante, sem a necessidade de abrir o circuito do ventilador. Volumes maiores de surfactante (em geral $4 \mathrm{ml} / \mathrm{kg}$ dos preparados a $25 \mathrm{mg} / \mathrm{ml}$ ) apresentam melhor distribuição do que a obtida com surfactantes mais concentrados.

\section{Complicações do tratamento}

Uma série de eventos de menor importância, e sem repercussão em longo prazo, podem estar associados à técnica de administração de surfactante em bolo, particularmente em volumes maiores. Estes eventos, que incluem cianose transitória, aumento de $\mathrm{paCO}_{2}$, taqui ou bradicardia e refluxo do surfactante para o circuito do ventilador, entre outros, podem ser evitados, ou são corrigidos sem dificuldades, através da administração do surfactante, utilizando-se técnica adequada e pessoal experiente. A elevação da $\mathrm{FiO}_{2} 10 \%$ acima da necessidade do recém-nascido no momento do tratamento, o uso de porta injetora lateral, ou cânula com dispositivo de infusão, associado a uma velocidade relativamente (mas, não excessivamente) lenta de infusão, com monitorização contínua da saturação de oxigênio, pode evitar ou minimizar estes eventos, que talvez não mereçam ser denominados de "complicações" associadas ao uso do surfactante.

Uma complicação verdadeira do tratamento com surfactante, embora rara, e de maior gravidade, por estar associada à alta morbidade e mortalidade, é a hemorragia pulmonar.

Vários autores relataram um aumento na incidência de hemorragia pulmonar, tanto após tratamento com surfactante sintético como com o natural, sendo um pouco mais elevado no último ${ }^{41}$. Ela ocorre várias horas após o tratamento, tendo sido feita a associação entre sua ocorrência e o aumento do fluxo esquerdo-direito através do canal $\operatorname{arterial}^{42}$.

\section{O que ainda não está bem determinado \\ O papel dos novos surfactantes}

Embora a composição do surfactante pulmonar seja bastante complexa, os principais elementos responsáveis pela sua função podem ser reduzidos a 4 componentes: a fosfatidilcolina (o principal elemento atuante na tensão superficial), o fosfatidilglicerol, e as proteínas lipossolúveis SP-B e SP-C. Estas duas últimas têm funções específicas no surfactante, e sua ausência implica em grande perda de função, indicando que elas devem estar presentes no produto final. Ambas as proteínas são encontradas nos surfactantes comerciais, obtidos por extração lipídica de pulmões de mamíferos, embora em menor quantidade do que a encontrada no surfactante natural. 
Em vista da importância fundamental que estas proteínas têm na função do surfactante pulmonar, novos produtos foram desenvolvidos, embora ainda não disponíveis comercialmente, com o objetivo de desenvolver sinteticamente as proteínas do surfactante sem a necessidade de extração animal. Uma preocupação com o uso da SP-C natural é o seu grau de pureza e a tendência à agregação, que se observa na sua extração ${ }^{43}$.

Um surfactante baseado em uma forma recombinante da SP-C humana modificada (rSP-C) foi recentemente desenvolvido e testado (Venticute ${ }^{\circledR}$, BYK Gulden Kenstanz, Alemanha). A SP-C humana é constituída por dipalmitoiilato de cistina, nas posições 4 e 5 , que na molécula de rSP$\mathrm{C}$ foram substituídos por fenilalanina. Essa mudança na estrutura molecular não modificou a função final avaliada in vivo ${ }^{43-45}$. Em um modelo de animal prematuro, a função deste novo surfactante foi similar à do surfactante natural de ovelha (que contém SP-B e SP-C) na dose de $100 \mathrm{mg} / \mathrm{kg}$, avaliado a partir de parâmetros de mecânica respiratória (pressão ventilatória necessária para se obter um volume corrente de 6 a $8 \mathrm{ml} / \mathrm{kg}$, complacência dinâmica e capacidade residual funcional) e gasométricos.

O novo surfactante foi bastante efetivo em dois modelos bem estabelecidos de imaturidade pulmonar, o coelho e o cordeiro prematuro. A função pulmonar em cordeiros prematuros, depois do tratamento com SP-C ou surfactante de ovelha, foi equivalente, exceto por uma pequena diferença na oxigenação ${ }^{46}$. Em coelhos prematuros, a resposta ao tratamento com ambos surfactantes foi semelhante, quando os animais foram ventilados com pressão expiratória final positiva (PEEP) de $3 \mathrm{~cm} / \mathrm{H}_{2} \mathrm{O}^{46}$.

Também utilizando-se modelos animais, foi observado que tanto a dexametasona quanto inibidores da fosfatodiesterase potencializam a ação do surfactante com rSP-C $\mathrm{C}^{47,48}$, e que seu uso em um modelo animal de hipersensibilidade não resultou em um aumento de risco para reação anafilática $^{49}$.

Recentemente, adultos com SDRA foram tratados com rSP-C, apresentando melhora da troca gasosa, menor necessidade de suporte ventilatório e melhor sobrevida ${ }^{50}$.

Estudos clínicos fase III, incluindo adultos com SDRA, estão atualmente sendo realizados.

Outro surfactante sintético desenvolvido recentemente é o KL-4 (Surfaxin ${ }^{\circledR}$, Discovery Laboratories, Daylestown, Estados Unidos), que contém um peptídio de 21 unidades (KLLLLKLLLLKLLLLKLLLLK, onde $\mathrm{K}=$ lisina e $\mathrm{L}=$ leucina), que mimetiza as propriedades da SP-B e fosfolípides (fosfatidilcolina e fosfatidilglicerol). Este novo surfactante mostrou ser eficaz na melhora da troca gasosa em modelos experimentais ${ }^{51} \mathrm{e}$ em recém-nascidos com SDR, tratados até 4 horas após o nascimento ${ }^{52}$.

Mais estudos randomizados fase III são necessários antes da utilização clínica de rotina destes novos surfactantes.

\section{Recrutamento alveolar e o tratamento com surfactan- te exógeno}

Vários estudos recentes sugerem que o recrutamento alveolar realizado através do aumento do pico de pressão inspiratória (PIP), durante a administração do surfactante, melhora os efeitos do tratamento ${ }^{53-55}$.

Considerando-se que o prematuro deficiente de surfactante tem uma tendência ao colabamento alveolar, com uma redução da capacidade residual funcional, as manobras de recrutamento realizadas antes da instilação de surfactante permitiriam a aeração das partes colabadas do pulmão, resultando em uma melhor distribuição e melhor resultado, obtido a partir do surfactante administrado.

Em coelhos deficientes de surfactante, obtidos por lavagem bronco-alveolar, submetidos à ventilação mecânica com PEEP $=1 \mathrm{cmH}_{2} \mathrm{O}$, o aumento da pressão inspiratória em 8 a $9 \mathrm{cmH}_{2} \mathrm{O}$, iniciado 2 minutos antes e mantidos até 4 minutos após o tratamento, resultou em melhora da capacidade residual funcional, do volume corrente, da complacência dinâmica e de parâmetros gasométricos ${ }^{53}$.

Por outro lado, partindo-se de uma situação mais fisiológica de uso de PEEP mais elevado $\left(3 \mathrm{cmH}_{2} \mathrm{O}\right)$, as manobras de recrutamento não levaram a uma melhor performance de mecânica respiratória ou gasométrica.

Também utilizando um modelo experimental de deficiência de surfactante por lavagem pulmonar, as manobras de recrutamento resultaram em uma distribuição mais homogênea do surfactante, com melhora da ventilação alveolar e da oxigenação $0^{55}$.

Por outro lado, é bem determinado que o uso de pressão expiratória mais elevada no momento do tratamento com surfactante resulta, pelo menos em teoria, em um melhor recrutamento alveolar, melhorando a função pulmonar e mantendo o surfactante em sua forma ativa, quando comparado à ausência, ou aos níveis baixos de PEEP ${ }^{56,57}$. Também é estabelecido que o uso de volumes correntes elevados resulta em maior conversão de surfactante para formas inativas $^{22}$ e maior lesão pulmonar, o que poderia predispor o pulmão prematuro à evolução para displasia broncopulmonar.

Finalmente, deve ser levado em consideração que, na fisiopatologia da SDR, as áreas hipoventiladas podem não estar necessariamente atelectasiadas, mas sim, preenchidas por líquido e proteínas, que não resultaria em melhora com as manobras de recrutamento.

Desta forma, no momento não é possível avalizar estratégias de recrutamento alveolar baseadas no aumento do volume corrente, secundárias a elevados picos de pressão inspiratória, em vista dos efeitos indesejados de longo prazo, secundários à possível lesão pulmonar induzida por este tipo de estratégia, os quais ultrapassariam qualquer efeito positivo da distribuição do surfactante, com melhoras imediatas da oxigenação e da mecânica respiratória.

Deve ainda ser ressaltado que o recrutamento pulmonar moderado, obtido com o uso de PEEP acima de níveis 
fisiológicos, pode melhorar a distribuição e a resposta do tratamento com surfactante.

\section{Uso de surfactante em outras afecções que não a SDR}

\section{Sindrome de aspiração meconial (SAM)}

O mecônio é um conhecido inibidor da atividade do surfactante pulmonar. Além de suas características obstrutivas e inflamatórias, o mecônio determina um aumento da tensão superficial, que resulta em múltiplo colabamento alveolar, com piora da função pulmonar já comprometida. Este efeito inibitório do mecônio sobre a função do surfactante pode ser revertido através do aumento da concentração deste último, e se constitui na base do tratamento com surfactante na síndrome de aspiração meconial.

Sun e colaboradores estudaram a função pulmonar de coelhos recém-nascidos maduros após aspiração de mecônio, após o tratamento com surfactante porcino na dose de $200 \mathrm{mg} / \mathrm{kg}$, observando melhora dos valores de complacência dinâmica e melhor aeração pulmonar no grupo trata$\mathrm{do}^{58}$. Em estudo posterior, esse mesmo autor comparou os efeitos da administração precoce ou tardia de surfactante exógeno (SE), observando melhora da troca gasosa, da complacência pulmonar, da expansão alveolar e menor lesão pulmonar em animais tratados, independentemente do tempo de administração do SE. Houve, ainda, menor necessidade de oxigenação e pressão média de vias aéreas, menor formação de membranas hialinas, menos edema intra-alveolar e menor influxo de neutrófilos para os espaços intra-alveolares.

Existem poucos estudos controlados avaliando o uso do SE, para tratamento da SAM; além disso, os que existem foram realizados com pequeno número de pacientes e têm resultados conflitantes. Halliday tratou 54 recém-nascidos com SAM com surfactante porcino e obteve pequena melhora na oxigenação. Quarenta e quatro por cento dos recém-nascidos não apresentaram nenhuma melhora, 20\% tiveram pneumotórax e $19 \%$ morreram. Dentre os sobreviventes, $18 \%$ desenvolveram pneumopatia crônica ${ }^{59}$. Lotze e colaboradores, num estudo multicêntrico, avaliaram o uso de surfactante bovino em uma população de 328 recémnascidos a termo, com grave insuficiência respiratória, sendo que $21 \%$ destes tinham SAM. Não houve diferença na mortalidade, nos dias de suplementação com oxigênio, na duração da ventilação mecânica, ou no tempo de internação, quando comparados os grupos tratados ou não trata$\operatorname{dos}^{60}$.

Apenas um estudo controlado e randomizado foi realizado, para avaliar o uso do SE na terapia da SAM. Vinte recém-nascidos com SAM foram tratados com SE em infusão contínua por 20 minutos. Melhora significante na oxigenação ocorreu após período de 6 a 12 horas, principalmente após doses adicionais de surfactante. $\mathrm{O}$ autor recomenda mais estudos clínicos antes do uso rotineiro de SE para tratamento da $\mathrm{SAM}^{61}$.

\section{Pneumonia}

A pneumonia extensa pode levar a uma disfunção do surfactante, tanto por um processo de inibição secundário ao extenso processo inflamatório e edema, que acompanha o processo de base, como por anormalidade nas frações fosfolipídicas e protéicas, que foram descritas associadas a uma variedade de patógenos, incluindo bactéria, vírus e fungos ${ }^{62}$.

Relatos de tratamento de pneumonia com surfactante em humanos são raros, e estudos controlados não são disponíveis. Em prematuros com falência respiratória secundária à pneumonia, o surfactante se mostrou seguro ${ }^{63}$, com melhora da hipoxemia em adultos ${ }^{64}$. De maneira semelhante, em recém-nascidos infectados pelo Streptococcus do grupo B e tratados com surfactante exógeno, observou-se uma melhora da oxigenação uma hora após o tratamento ${ }^{65}$.

A imunogenicidade e a atividade moduladora do surfactante exógeno também necessitam de maior investigação antes da liberação do surfactante para uso rotineiro na pneumonia grave.

\section{Hérnia diafragmática congênita}

Existem evidências da experimentação animal ${ }^{66} \mathrm{e} e m$ humanos ${ }^{67}$ que sugerem melhora da função pulmonar e da oxigenação com o uso de surfactante exógeno na hérnia diafragmática congênita, com melhora da sobrevida. No entanto, a falta de estudos randomizados e controlados, de maior casuística, não permite assumir que os efeitos benéficos, em curto prazo, se reflitam em melhora da mortalidade ou morbidade em longo prazo. Isto é particularmente importante, se levarmos em consideração que a hérnia diafragmática congênita é uma doença que continua com elevadas taxas de mortalidade, a despeito de intervenções extremas, como a terapia com óxido nítrico e a oxigenação por membrana extracorpórea.

\section{Bronquiolite}

A bronquiolite acomete crianças abaixo de 2 anos de idade e fora do período neonatal. É uma infecção causada principalmente pelo vírus sincicial respiratório. $\mathrm{O}$ achado histopatológico típico inclui lesão alveolar e bronquiolar terminal, envolvendo a membrana alvéolo-capilar e os pneumócitos, com lesão obstrutiva das pequenas vias aéreas.

A lesão dos pneumócitos tipo II leva a uma disfunção qualitativa do surfactante ${ }^{68}$, que contribui para o colapso alveolar e o aumento da permeabilidade capilar, piorando, agora por inativação, a disfunção do surfactante.

$\mathrm{O}$ tratamento com surfactante foi proposto em vista de sua atividade estabilizadora dos bronquíolos terminais e alvéolos e da melhora da troca gasosa. Em um estudo randomizado, o uso de Curosurf ${ }^{\circledR}$ resultou em melhora da oxigenação, redução do tempo de ventilação mecânica e do tempo de permanência na UTI ${ }^{69}$. 


\section{Síndrome do Desconforto Respiratório tipo Agudo (SDRA)}

Em 1996, Anzueto et al. realizaram um ensaio clínico randomizado com 725 pacientes adultos, com SDRA induzida por sepse, para avaliar a eficácia do surfactante por aerossol ${ }^{70}$. Não houve diferença estatisticamente significante na sobrevida com 30 dias pós-tratamento, no tempo de permanência na UTI, na duração da ventilação mecânica, ou no desfecho fisiológico (oxigenação).

$\mathrm{O}$ artigo de Anzueto et al. ${ }^{70}$ foi comentado num editorial por Matthay ${ }^{71}$. As possíveis explicações para o não funcionamento do surfactante aerolizado na SDRA são:

- a quantidade de surfactante que atingiu os alvéolos periféricos foi de apenas 5\% da dose administrada;

- a preparação de surfactante sintético utilizada, Exosurf, não tem o componente protéico. Os surfactantes que contêm proteínas podem reduzir a tensão superficial mais rapidamente e são mais resistentes aos inibidores, como as proteínas plasmáticas;

- outra razão possível para a falha da terapia com surfactantes é que, apesar das semelhanças, SDRA e SDR-RN são fundamentalmente diferentes. A lesão inflamatória da SDRA, que comumente progride para a destruição fibrótica do pulmão, pode não melhorar com o surfactante;

- a maioria dos pacientes da casuística do ensaio clínico de Anzueto et al. morreu de sepse e falência de múltiplos órgãos, e não de insuficiência respiratória. Com a terapia com surfactantes, não seria de se esperar que reduzisse as mortes dos pacientes com SDRA devido a infecções generalizadas, ou disfunção de órgãos.

\section{Considerações finais}

A terapêutica com surfactante exógeno é bastante efetiva e simplificou, de maneira profunda, o tratamento de recém-nascidos com síndrome do desconforto respiratório. A terapêutica é efetiva porque alia a correção da deficiência quantitativa primária de surfactante (a principal responsável pela falência respiratória) com um metabolismo favorável. No pulmão não lesado, uma única dose é suficiente para melhorar a função pulmonar até o recém-nascido se tornar capaz de sintetizar uma quantidade suficiente de surfactante endógeno. Com o estabelecimento da lesão pulmonar, a função do surfactante utilizado no tratamento se deteriora, e a habilidade do pulmão prematuro de produzir surfactante pode estar diminuída. Embora o tratamento com surfactante não pareça reduzir a incidência de DBP, este achado resulta de uma maior sobrevida de recém-nascidos, que sem este tratamento morreriam, e que são de risco elevado para o desenvolvimento da DBP. As interações entre as funções do surfactante e as variáveis do ventilador são áreas importantes, nas quais a resposta pulmonar geral pode ser melhorada. O uso de PEEP adequada, de baixo volume corrente, e o cuidado para se evitar a hiperventilação melhoram a função do surfactante e minimizam a lesão pulmonar. Embora os surfactantes produzidos a partir de pulmões de animais sejam comparáveis em relação à sua eficácia, eles são caros e não são padronizados. Uma meta para o futuro é o desenvolvimento de surfactantes de menor custo e mais uniformemente consistentes.

\section{Referências bibliográficas}

1. Von Neergaard K. Neue auffassungen uber einen Grundbergriff der Atemmechanik. Die retraktion-skraft der lunge, abhangig von der Oberflachenspannung in den Alveolen. Z Gesamte Exp Med 1929;66:373.

2. Gruenwald P. Surface tension as a factor in the resistance of neonatal lungs to aeration. Am J Obstet Gynecol 1947;53:9961001.

3. Avery ME, Mead J. Surface properties in relation to atelectasis and hyaline membrane disease. Am J Dis Child 1959;97:517-23.

4. Fujiwara T, Maeta H, Chida S, Morita T, Watabe Y, Abe T. Artificial surfactant therapy in hyaline membrane disease. Lancet 1980;1:55-9.

5. Soll RF, Blanco F. Natural surfactant extract versus synthetic surfactant for neonatal respiratory distress syndrome [review]. Cochrane Database of Syst Rev 2001;(2):CD000144.

6. Soll RF, Morley CJ. Prophylactic versus selective use of surfactant in preventing morbidity and mortality in preterm infants [review]. Cochrane Database of Syst Rev 2001;(2); CD000511.

7. Soll RF. Multiple versus single dose natural surfactant extract for severe neonatal respiratory distress syndrome [review]. Cochrane Database of Syst Rev 2000;(2):CD000141.

8. Shelley SA, Paciga JE, Balis JU. Lung surfactant phospholipids in different animal species. Lipids 1984;19:857-62.

9. Goerke J. Pulmonary surfactant: functions and molecular composition. Biochim Biophys Acta 1998;1408:79-89.

10. LeVine AM, Kurak KE, Wright JR, Watford WT, Bruno MD, Ross GF, et al. Surfactant protein A (SP-A) binds group B streptococcus enhancing phagocytosis and clearance from lungs of surfactant protein A deficient mice. Am J Respir Cell Mol Biol 1999;20(2):279-86

11. Cockshutt AM, Weitz J, Possmeyer F. Pulmonary surfactantassociated protein A enhances the surface activity of lipid extract surfactant and reverses inhibition by blood proteins in vitro. Biochemistry 1990;29:8424-9.

12. Nogee LM, Garnier G, Dietz HC, Singer L, Murphy AM, deMello DE, et al. A mutation in the surfactant protein $B$ gene responsible for fatal respiratory disease in multiple kindreds. J Clin Invest 1994;93:1860-3.

13. Nogee LM, DeMello DE, Dehner LP, Colten HR. Deficiency of pulmonary surfactant protein $B$ in congenital alveolar proteinosis. N Engl J Med 1993;328:406-10.

14. Suzuki Y, Fujita Y, Kogishi K. Reconstitution of tubular myelin from synthetic lipids and proteins associated with pig pulmonary surfactant. Am Rev Respir Dis 1989;140:75-81.

15. Glasser SW, Burhans MS, Korfhagen TR, Sly PD, Ross GF, Ikegami M, et al. Generation of an SP-C Deficient mouse by targeted gene inactivation. Am J Respir Crit Care Med 2000;161:A43.

16. Crouch EC. Structure, biologic properties and expression of surfactant protein D (SP-D). Biochim Biophys Acta 1998; 1408:278-89. 
17. Gail DB, Steinkamp H, Massaro D. Interspecies variation in lung lavage and tissue saturated phosphatidylcholine. Respir Physiol 1978;33:289-97.

18. Pre J, Perret G, Bladier D. Lecithin content estimate of human alveolar lining layer: comparison with mouse, rat and rabbit. Comp Biochem Physiol 1983;76A:393-5.

19. Rebello CM, Jobe AH, Eisele JW, Ikegami M. Alveolar and tissue surfactant pool size in humans. Am J Respir Crit Care Med 1996;154:625-8.

20. Hallman M, Merritt TA, Pohjavuori M, Gluck L. Effect of surfactant substitution on lung effluent phospholipids turnover, pool size and the relationship to severity of respiratory failure. Pediatr Res 1986;20:1228-35.

21. Jobe AH, Ikegami M, Sarton-Miller I. Surfactant metabolism of newborn lamb lungs in vivo. J Appl Physiol 1980;49:1091-8.

22. Ito Y, Ruud AWV, Yao LJ, MacCaig LA, Bartlett AJ, Lewis JF. Ventilation strategies affect surfactant aggregate conversion in acute lung injury. Am J Respir Crit Care Med 1997;155:493-9.

23. Ikegami M, Ikegami M. Surfactant metabolism. Semin Perinatol 1993; $17: 233-40$

24. Ikegami, M. Surfactant inactivation. In: Boyton BR, Carlo WA, Jobe AH, editores. New Therapies for Neonatal Respiratory Failure. New York: Cambridge University Press; 1994.p.36-48.

25. Seeger W, Gunther A, Thede C. Differential sensitivity to fibrinogen inhibition of SP-C vs SP-B based surfactants. Am J Physiol 1992;261:L286-91.

26. Yukitake K, Brown CL, Schlueter MA, Clements JA, Hawgood S. Surfactant apoprotein A modifies the inhibitory effect of plasma proteins on surfactant activity in vivo. Pediatr Res 1995; 37:21-5.

27. Rebello CM, Ikegami M, Polk DH, Jobe AH. Postnatal lung responses and surfactant function after fetal or maternal corticosteroid treatment of preterm lambs. J Appl Physiol 1996; 80:1674-9.

28. Shardonofsky FR, Sato J, Bates JHT. Quasi-static pressurevolume hysteresis in the canine respiratory system in vitro. J Appl Physiol 1990;68:2230-6.

29. Goldsmith LS, Greenspan JS, Rubenstein SD, Wolfson MR, Shaffer TH. Immediate improvement in lung volume after exogenous surfactant: alveolar recruitment versus increased distention. J Pediatr 1991;119(3):424-8.

30. Alexander J, Milner AD. Lung volume and pulmonary blood flow measurements following exogenous surfactant. Eur J Pediatr 1995;154:392-7.

31. Hamdan AH, Shaw NJ. Changes in pulmonary artery pressures during the acute phase of respiratory distress syndrome treated with three different types of surfactant. Pediatr Pulmonol 1998;25:191-5.

32. Stevens TP, Blennow M, Soll RF. Early surfactant administration with brief ventilation vs selective surfactant and continued mechanical ventilation for preterm infants with or at risk for RDS [review]. Cochrane Database of Syst Rev 2002;(2): CD003063.

33. Jobe AH, Ikegami M. Protein permeability abnormalities in the preterm. In: Effros RM, Chang HK, editores. Lung Biology in Health \& Disease. Fluid and solute transport in the airspaces of the lung. New York: Mascel Dekker Inc.; 1994.p.335-55.

34. Ikegami M, Adams FH, Towers B, Osher AB. The quantity of natural surfactant necessary to prevent the respiratory distress syndrome in premature lambs. Pediatr Res 1980;14:1082-5.

35. King RJ, Clements JA. Surface active materials from dog lung. II Composition and physiological correlation. Am J Physiol 1972;223:715-9.

36. Fujiwara T. Surfactant replacement in neonatal RDS. In: Robertson B, Van Golde LMG, Batemburg JJ, editores. Pulmonary surfactant. Amsterdam: Elsevier Science Publishers; 1984.p.480503.
37. Henry MD, Rebello CM, Ikegami M, Jobe AH, Langenback EG, Davis JM. Ultrasonic nebulized in comparison with instilled surfactant treatment of preterm lambs. Am J Respir Crit Care Med 1996;154:366-75.

38. Jobe AH, Ikegami M, Jacobs H, Jones S. Surfactant and pulmonary blood flow distributions following treatment of premature lambs with natural surfactant. J Clin Invest 1984;73:848-56.

39. Broadbent R, Fok TF, Dolovich M. Chest position and pulmonary deposition of surfactant in surfactant depleted rabbits. Arch Dis Child Fetal Neonatal Ed 1995;72:F84-F89.

40. Zola EM, Gunkel JH, Chan RK, Lim MO, Knox I, Feldman BH, et al. Comparison of three dosing procedures for administration of bovine surfactant to neonates with respiratory distress syndrome. J Pediatr 1993;122:453-9.

41. Raju TNK, Langenberg P. Pulmonary hemorrhage and exogenous surfactant therapy: a metaanalysis. J Pediatr 1993;123:603-10.

42. Garland J, Buck R, Weinberg M. Pulmonary hemorrhage risk in infants with a clinically diagnosed patent ductus arteriosus: a retrospective cohort study. Pediatrics 1994;94:719-23.

43. Hafner D, Germann PG, Hauschke D. Comparison of rSP_C surfactant with natural and synthetic surfactants after late treatment in a rat model of acute respiratory distress syndrome. Br J Pharmacol 1998;124:1083-90.

44. Spragg RG, Smith RM, Harris K, Lewis J, Hafner D, Germann P. Effect of recombinant SP-C surfactant in a porcine lavage model of acute lung injury. J Appl Physiol 1999;88:674-81.

45. Beers MF, Fisher AB. Surfactant protein C: a review of its unique properties and metabolism. Am J Physiol 1992;263: 151-60.

46. Davis AJ, Jobe AH, Hafner D, Ikegami M. Lung function in premature lambs and rabbits with a recombinant SP-C surfactant. Am J Respir Crit Care Med 1998;157:553-9.

47. Hafner D, Germann PG. Additive effects of phosphodiesterase4 inhibition on effects of rSP-C surfactant. Am J Respir Crit Care Med 2000;161(5):1495-500.

48. Hafner D, Germann PG. Dexamethasone enhances the activity of rSP-C surfactant but not of exosurf in a rat model of the acute lung injury. J Pharmacol Toxicol Methods 1999;42(1):39-48.

49. Germann PG, Kemkowski J, Hauschke D, Steinhilber W, Hafner D. Intratracheally applied rSP-C surfactant exhibits no anaphylactic shock reactions in a guinea pig model of acute lung hypersensitivity. Exp Toxicol Pathol 1999;51(6):495-505.

50. Walmrath D, De Vaal JB, Bruining HA, Kilian JG, Papazian L, Hohlfeld J, et al. Treatment of ARDS with a recombinant SP-C (rSP-C) based synthetic surfactant. Am J Respir Crit Care Med 2000;161:A379.

51. Cochrane CG, Revak SD. Surfactant lavage treatment in a model of respiratory distress syndrome. Chest. 1999 Jul;116 Suppl 1:85-86.

52. Cochrane CG, Revak SD, Merritt TA, Heldt GP, Hallman M, Cunningham MD, et al. The efficacy and safety of KL4-surfactant in preterm infants with respiratory distress syndrome. Am J Respir Crit Care Med 1996;153(1):404-10.

53. Krause M, Olsson T, Law AB, Parker RA, Lindstrom DP, Sundell HW, et al. Effect of volume recruitment on response of surfactant treatment in rabbits with lung injury. Am J Respir Crit Care Med 1997;156:862-6.

54. Krause MF, Hoehn T. Enhancement of surfactant effect by a mechanical volume recruitment maneuver depends on the lungs preexisting distension. Biol Neonate 1998;73:320-9.

55. Krause MF, Jakel C, Haberstroh J, Schulte-Monting J, Leititis J, Orlowska-Volk M. Alveolar recruitment promotes homogeneous surfactant distribution in a piglet model of lung injury. Pediatr Res 2001;50:34-43.

56. Michna J, Jobe AH, Ikegami M. Positive end-expiratory pressure preserves surfactant function in preterm lambs. Am J Respir Crit Care Med 1999;160:634-9. 
57. Ogawa A, Brown CL, Schlueter MA, Benson BJ, Clements JA, Hawgood S. Lung function, surfactant apoprotein content, and level of PEEP in prematurely delivered rabbits. J Appl Physiol 1994;77:1840-9.

58. Sun B, Cursted T, Song GW, Robertson B. Surfactant improves lung function and morphology in newborn rabbits with meconium aspiration. Biol Neonate 1993;63:96-104.

59. Halliday HL, Speer CP, Robertson B. Treatment of severe meconium aspiration syndrome with porcine surfactant. Eur $\mathbf{J}$ Pediatr 1996;155:1047-51.

60. Lotze A, Mitchell BR, Bulas DI, Zola EM, Shalwitz RA, Gunkel JH. Multicenter study of surfactant (beractant) use in the treatment of term infants with severe respiratory failure. J Pediatr 1998;132:40-47.

61. Findlay RD, Taeusch HW, Walter FJ. Surfactant replacement therapy for meconium aspiration syndrome. Pediatrics 1996;97:48-52.

62. Baughman RP, Sternberg RI, Hull W, Buchsbaum JA Whitsett J. Decreased surfactant protein A in patients with bacterial pneumonia. Am Rev Respir Dis 1993;147:653-7.

63. Brehmer U, Jorch G. Surfactant therapy in severe neonatal respiratory failure - multicenter study III. Surfactant therapy in 41 premature infants under 34 weeks with suspected congenital infection (case-control analysis). Klin Padiatr 1993;205:78-82.

64. Mikawa K, Maekawa N, Nishina K, Takao Y, Yaku H, Obara H. Selective intrabronchial instillation of surfactant in a patient with pneumonia: a preliminary report. Eur Respir J 1993;6:1563-6.

65. Herting E, Gefeller O, Land M, van Sonderen L, Harms K, Robertson B. Surfactant treatment of neonates with respiratory failure and group B streptococcal infection. Pediatrics 2000;106(5):957-64.
66. Glick PL, Stannard VA, Leach CL, Rossman J, Hosada Y, Morin FC, et al. Pathophysiology of congenital diaphragmatic hernia II: the fetal lamb CDH model is surfactant deficient. J Pediatr Surg 1992;27:382-8.

67. Glick PL, Stannard VA, Leach CL, Rossman J, Hosada Y, Morin FC, et al. Pathophysiology of congenital diaphragmatic hernia III: Exogenous surfactant therapy for the high risk neonate with CDH. J Pediatr Surg 1992;27:866.

68. Keer MH, Paton JY. Surfactant protein levels in severe respiratory syncytial virus infection. Am J Respir Crit Care Med 1999;159(4 Pt 1):1115-8.

69. Luchetti M, Casiraghi G, Valsecchi R, Galassini E, Marraro G. Porcine-derived surfactant treatment of severe bronchiolitis. Acta Anaesthesiol Scand 1998;42:805-10.

70. Anzueto A, Baughman RP, Guntupalli KK, Weg JG, Wiedemann HP, Raventos AA, et al. Aerosolized surfactant in adults with sepsis-induced acute respiratory distress syndrome. N Engl J Med 1996;334:1417-21.

71. Matthay MA. The acute respiratory distress syndrome. N Engl J Med 1996;334:1469-70.

Endereço para correspondência:

Dr. Celso M. Rebello

Av. Dr. Enéas de Carvalho Aguiar, 647

CEP 05403-900 - São Paulo, SP

Fone: (11) 3066.7360

E-mail: celsomr@dialdata.com.br 